\title{
HUMAN APOLIPOPROTEIN C1 TRANSGENESIS TO INHIBIT CETP AND TO ATTENUATE ATHEROSCLEROSIS IN HYPERCHOLESTEROLEMIC RABBITS
}

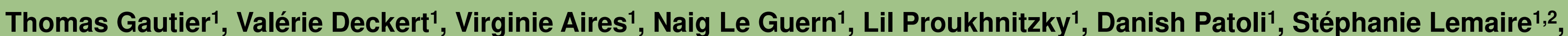
Guillaume Maquart ${ }^{1}$, Amandine Bataille ${ }^{1}$, Marion Xolin ${ }^{1}$, Charlène Magnani ${ }^{1}$, David Masson ${ }^{1,2}$, Erwana Harscoët ${ }^{3}$, Bruno Da Silva ${ }^{3,4}$, Louis-Marie Houdebine ${ }^{3}$, Geneviève Jolivet $^{3}$, Laurent Lagrost ${ }^{1,2}$.

${ }^{1}$ INSERM / University of Bourgogne Franche-Comté LNC UMR1231, LipSTIC LabEx, UFR Sciences de Santé, Dijon, France; ${ }^{2}$ University Hospital of Dijon, Dijon, France; ${ }^{3}$ UMR BDR, INRA, ENVA, Université Paris Saclay, Jouy en Josas, France; ${ }^{4}$ Laboratory of Developmental Biology, CNRS UMR7622, Université Pierre et Marie Curie, Paris, France.

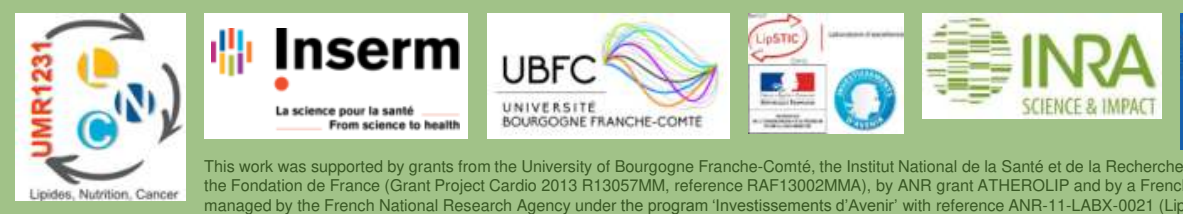

\section{OBJECTIVE}

Apolipoprotein (apo) $\mathrm{C} 1$ is a $6.6 \mathrm{kDa}$ protein associated with HDL and VLDL. ApoC1 alters triglyceride clearance by inhibiting lipolysis and VLDL uptake, and it also favors cholesterol accumulation in HDL, especially by inhibiting CETP. Apart from studies in mice, which lack CETP, the impact of apoC1 on atherosclerosis in animal models which, like humans, express CETP is not known. The objective of this study was to determine the net effect of human apoC1 on atherosclerosis in rabbits, a species with naturally high CETP activity but dysfunctional apoC1.

\section{GENERATION OF TRANGENIC RABBITS EXPRESSING HUMAN APOC1}

\section{Construct}

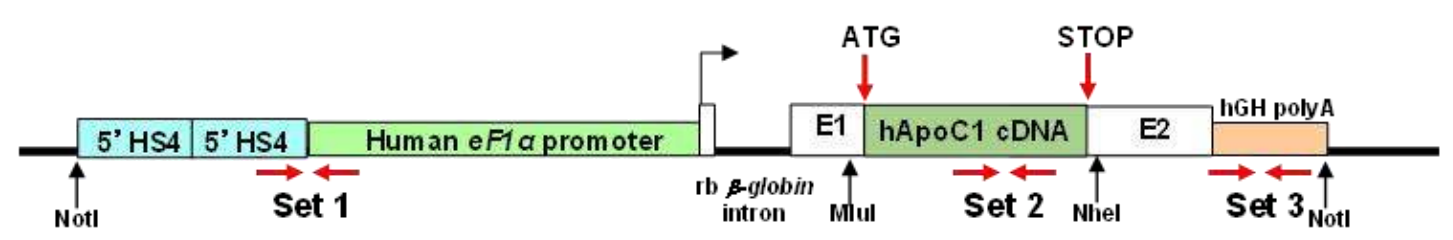

Gene expression pattern

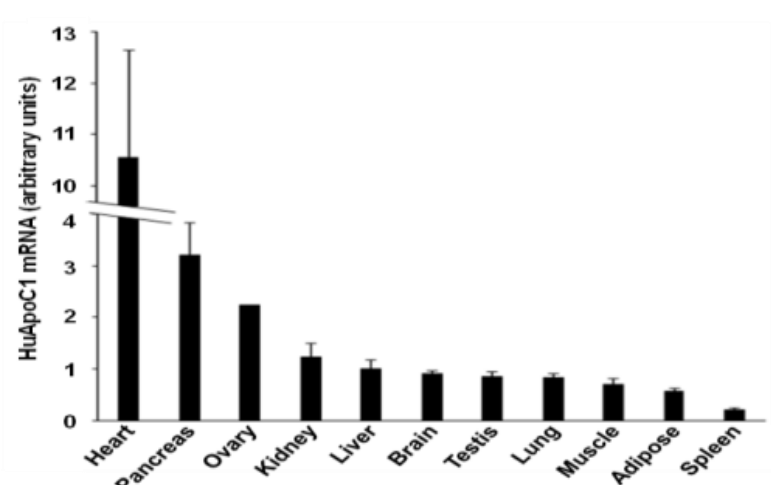

Plasma distribution of human ApoC1

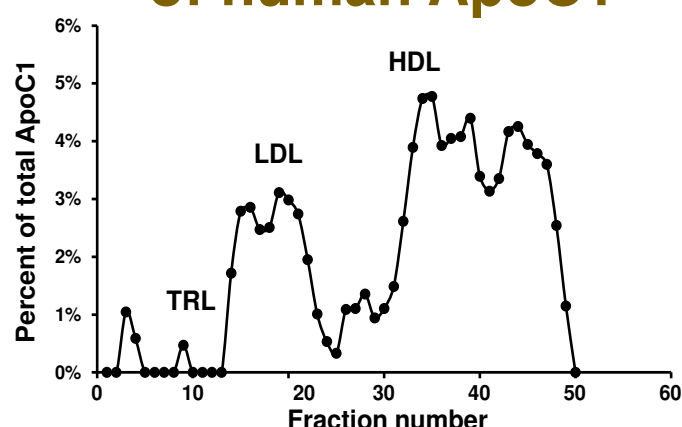

One transgenic line harboring 3 copies of the transgene was successfully established. The EF1a promoter led to ubiquitous expression of the transgene, with the highest levels in heart and pancreas. In plasma, most human apoC1 co-localized with HDL, with smaller amounts present in the low-density lipoprotein (LDL) as well as in the lipoprotein-free fraction

CHARACTERISTICS OF CHOW-FED RABBITS

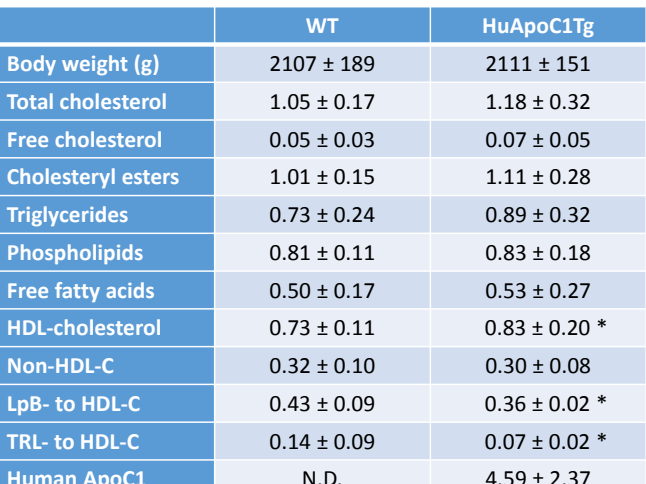

Cholesterol profile

HuApoC1Tg rabbits had higher HDL-C and lower LpB to HDL ratio than WT rabbits.

This protective lipid profile might relate to lower CETP activity in HuApoC1Tg rabbits. The decrease in specific CETP activity indicates that the expression of human ApoC1 successfully restores CETP inhibition in HuApoC1Tg rabbits.

\section{CHARACTERISTICS OF RABBITS ON A HC DIET}
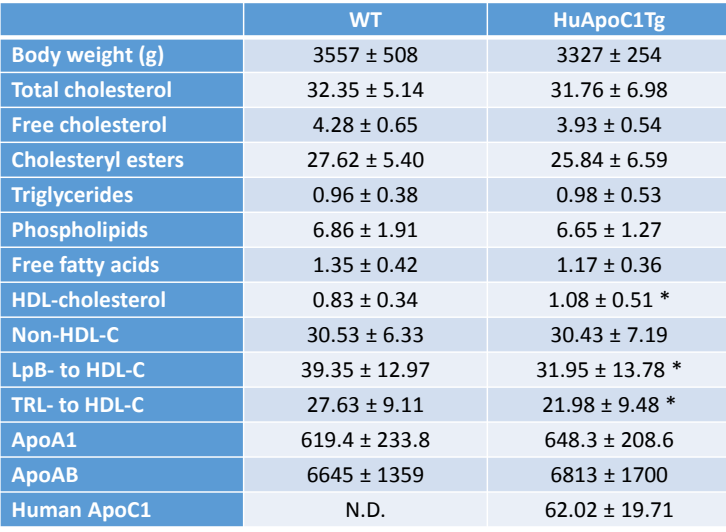

Cholesterol profile

After 8 weeks of a hypercholesterolemic diet (HC diet), HuApoC1Tg rabbits still had higher HDL cholesterol levels than WT rabbits.

$\mathrm{HDL}$ isolated from HuApoC1Tg rabbits showed lower ability to interact with CETP.

This translates into more protective lipid profile as reflected by the lower $\mathrm{LPB}$ to $\mathrm{HDL}$ ratio in HuApoC1Tg rabbits.

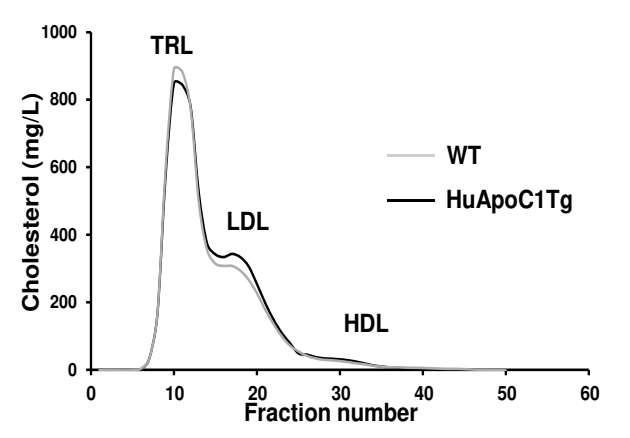

CETP activity

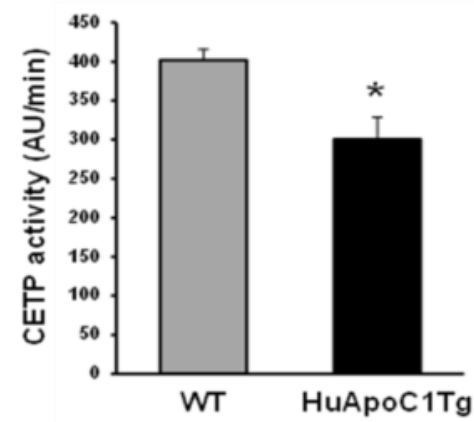

\section{HDL PARAMETERS IN RABBITS ON A HC DIET}

HDL size
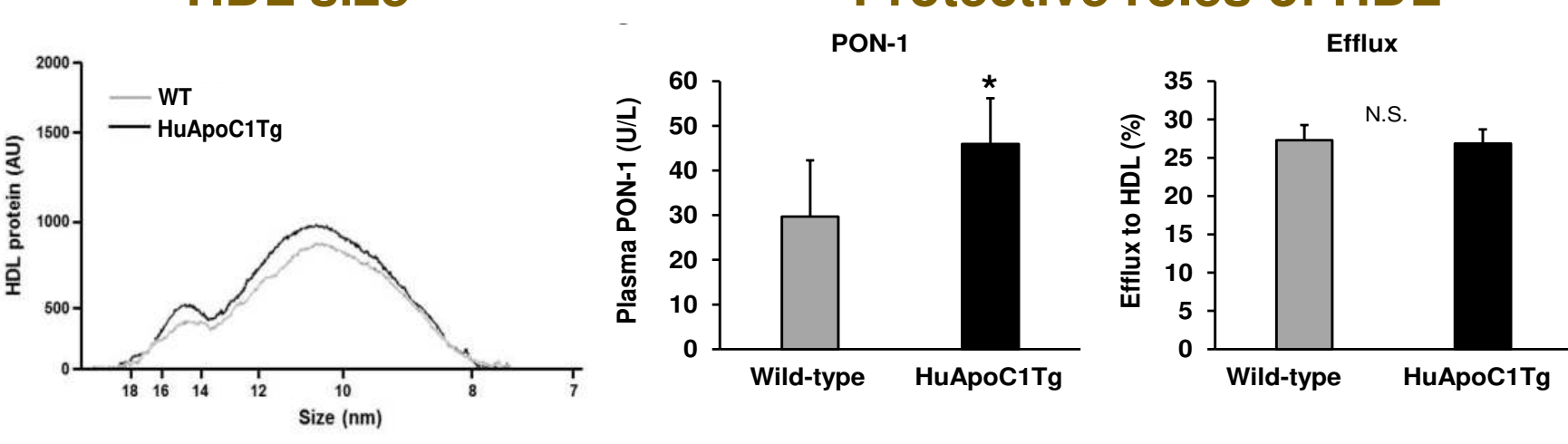

HuApoC1Tg rabbits displayed higher amounts of large-sized HDL.

PON-1 activity was significantly increased in HuApoC1Tg rabbits, together with a significant $16 \%$ decrease in the plasma levels of lipid oxidation marker 12(S) HODE (71.1 \pm 4.4 versus $84.0 \pm 10.0$ in HuApoC1Tg and WT rabbits, respectively, $\mathrm{p}<0.05)$.

ATHEROSCLEROSIS IN RABBITS ON A HC DIET
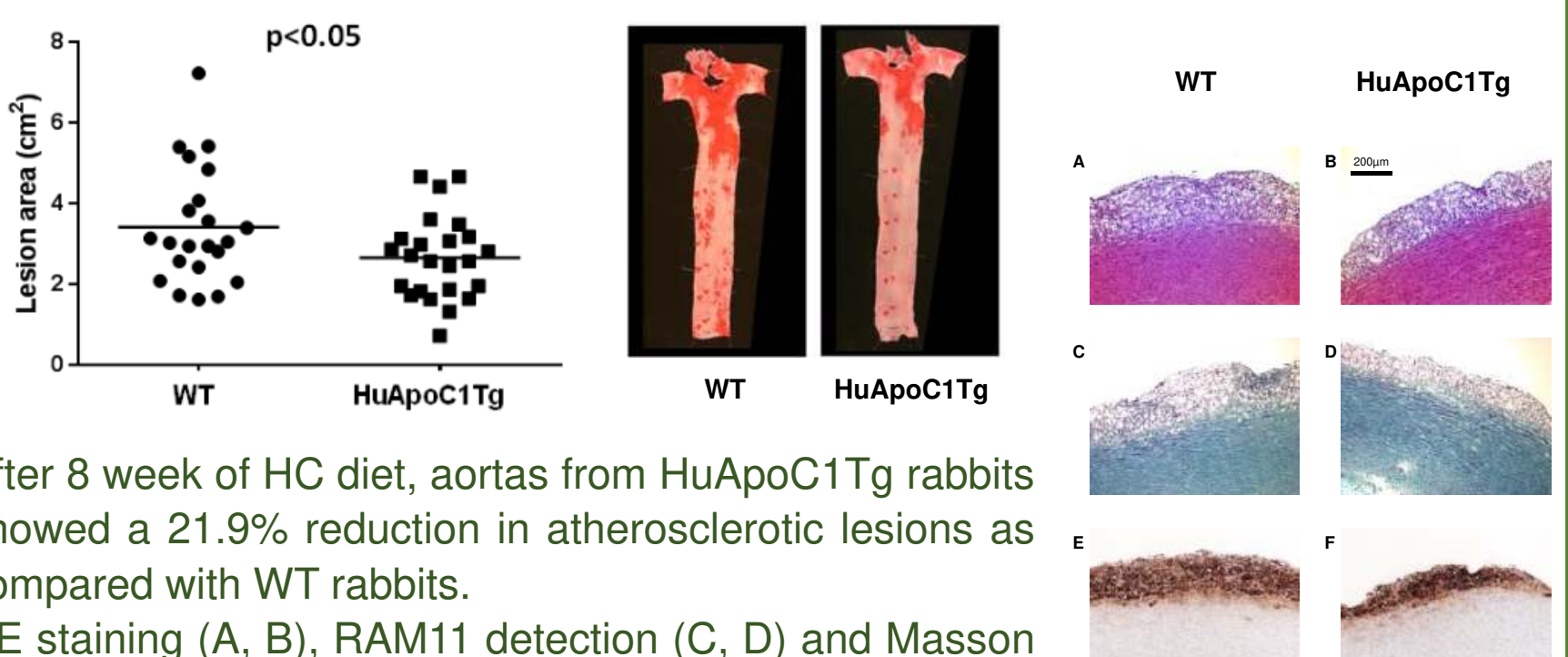

After 8 week of HC diet, aortas from HuApoC1Tg rabbits showed a $21.9 \%$ reduction in atherosclerotic lesions as compared with WT rabbits.

HE staining (A, B), RAM11 detection (C, D) and Masson Trichrome staining (E, F) of aortic lesions showed similar thickening of arterial intima and enrichment in macrophages and collagen fibers in both groups.

\section{CONCLUSION}

Expression of fully functional human apoC1 in transgenic rabbit plasma inhibits CETP activity, increases plasma HDL levels and functionality and consequently reduces the extent of atherosclerosis. ApoC1, as a natural and endogenous CETP inhibitor, arises as an alternative to pharmacological CETP inhibitors, which are still challenged in clinical studies. It emerges as a novel candidate to raise HDL levels and to fight against atherosclerosis in humans. 